\section{Radioiodine treatment and mortality in patients with hyperthyroidism}

Researchers in Finland have uncovered a link between radioiodine treatment for hyperthyroidism and increased mortality rates. Radioiodine is a common treatment for hyperthyroidism, yet its long-term safety has not been established. Furthermore, it is not known whether the increased mortality rates suggested by some studies are attributable to the treatment or the condition.

To investigate this question, Metso et al. compared mortality rates in hyperthyroid patients receiving radioiodine treatment $(n=2,793)$ with those in age- and sex-matched controls $(n=2,793)$ in a population-based cohort study.

Patients were followed until death, emigration, or the end of the study period, and the median follow-up was 9 years. The results revealed increased overall, cerebrovascular, and cancerspecific mortality rates in hyperthyroid patients treated with radioiodine, compared with controls (rate ratio $[\mathrm{RR}] 1.12,1.40$, and 1.29, respectively). Mortality rates were higher in older patients, those with nodular thyroid disease, and those receiving higher radioiodine doses. Mortality rates were not affected by antithyroid medication. Mortality was increased by radioiodinetreated hyperthyroidism (RR 1.56) and age (RR 1.10 per year), whereas the development of hypothyroidism reduced mortality (RR 0.52).

The authors conclude that the elevated cerebrovascular mortality is probably due to the hyperthyroidism itself rather than the treatment, whereas the radioiodine could have contributed to increased cancer mortality. These findings support the active treatment of hyperthyroidism with lifelong follow-up and monitoring of cardiovascular risk factors.

Original article Metso S et al. (2007) Increased cardiovascular and cancer mortality after radioiodine treatment for hyperthyroidism. J Clin Endocrinol Metab 92: 2190-2196

\section{Genetic variant associated with BMI and adiposity}

Obesity has a strong hereditary component, but little progress has been made in defining the molecular genetics involved. Frayling et al. used a genome-wide study to identify single nucleotide polymorphisms (SNPs) in the human FTO gene that are associated with type 2 diabetes, and found that this association might be mediated by BMI. They went on to study the association of an FTO-variant (the $A$ allele of SNP rs9939609) with BMI and overweight or obesity in more than 38,500 white European participants.

The average BMI was significantly higher in adults carrying the $A$ allele than in noncarriers (per-allele change $0.34-0.46 \mathrm{~kg} / \mathrm{m}^{2}, P<10^{-34}$ ). The presence of the $A$ allele also increased the risk of being overweight or obese (perallele odds ratio 1.18 and 1.31, respectively). These associations were stronger in homozygotes for the $A$ allele than in heterozygotes. Children who carried the $A$ allele did not have a higher birth weight, but they had a higher BMI by age 7 years and higher risk of childhood overweight and obesity, as compared with noncarriers. The increase of BMI was attributable to increased adiposity.

The authors conclude that a common variant of the FTO gene is associated with BMI and fat mass in white European children and adults. The finding of the first highly reproducible genetic association with obesity is an important first step.

Original article Frayling TM et al. (2007) A common variant in the FTO gene is associated with body mass index and predisposes to childhood and adult obesity. Science 316: 889-894

\section{Nonmelanoma skin cancer is associated with a lower risk of osteoporotic fractures}

Vitamin D deficiency is associated with an increased risk of osteoporosis and subsequent low-trauma fractures in elderly people. Sunlight exposure helps to maintain normal serum levels of 25-hydroxyvitamin D; however, ultraviolet $B$ radiation derived from sunlight increases the risk of skin cancer. Srikanth et al. assessed the effect of cumulative sun exposure on the occurrence of osteoporotic fractures by using nonmelanoma skin cancer (NMSC) as a biomarker of sun exposure levels.

Data were obtained from two Southern Tasmanian population-based registries: a record of incident fractures that occurred between 1997 and 2000 and a record of incident NMSC diagnosed between 1991 and 2000. The study group (fracture cohort) 